\title{
The secrets of T Pyxidis
}

\author{
I. UV observations ^ \\ R. Gilmozzi ${ }^{1}$ and P. Selvelli ${ }^{2}$ \\ 1 European Southern Observatory, Karl-Schwarzschild-Str. 2, 85748 Garching, Germany \\ e-mail: rgilmozz@eso.org \\ 2 INAF - Osservatorio Astronomico di Trieste, via Tiepolo 11, 34143 Trieste, Italy \\ e-mail: selvelli@oats.inaf.it
}

Received 9 September 2005 / Accepted 25 August 2006

\section{ABSTRACT}

\begin{abstract}
Aims. We study the UV spectral behavior of the recurrent nova T Pyx during 16 years of IUE observations.
Methods. We examined both the IUE line-by-line images and the extracted spectra in order to understand the reality and the origin of the observed spectral variations. We compare different extraction methods and their influence on the spectrum of an extended object. Results. The UV continuum of T Pyx has remained nearly constant in slope and intensity over this time interval, without any indication of long-term trends. The reddening determined from the UV data is $E_{B-V}=0.25 \pm 0.02$. The best single-curve fit to the dereddened UV continuum is a power-law distribution $\propto \lambda^{-2.33}$. The tail of this curve agrees well with the $B, V$, and $J$ magnitudes of T Pyx, indicating that the contribution of the secondary star is negligible. One peculiar aspect of T Pyx is that most emission lines (the strongest ones being those of CIV 1550 and HeII 1640) show substantial changes both in intensity and detectability, in contrast to the near constancy of the continuum. Several individual spectra display emission features that are difficult to identify, suggesting a composite spectroscopic system. We tentatively ascribe the origin of these transient emission features either to loops and jets from the irradiated secondary or to moving knots of the surrounding nebula that are (temporarily) projected in front of the system. The inspection of all IUE line-by-line images has led to the detection of emission spikes outside the central strip of the spectrum, which in some cases seem associated to known emission features in the (main) spectrum. A comparison with other ex-novae reveals a surprising similarity to the spectrum of the very-slow nova HR Del, whose white dwarf primary has a mass that is allegedly about one half that of T Pyx.
\end{abstract}

Key words. stars: novae, cataclysmic variables - ultraviolet: stars

\section{Introduction}

The five recorded outbursts of the recurrent nova (RN) T Pyx occurred in 1890, 1902, 1920, 1944, and 1966, with a mean recurrence time of $19 \pm 5.3 \mathrm{yrs}$ (Webbink et al. 1987, hereinafter WLTO). All outbursts were remarkably similar in photometric behavior and characterized by a speed class that was substantially slower than in other RNe, with $t_{2}=60^{\mathrm{d}}$ instead of a few days. We note that according to the classifications of PayneGaposchkin (1957) and Warner (1995) T Pyx should be considered "moderately fast" and not "extremely slow" as reported in previous works.

It is unfortunate that the orbital period of the binary system has not been clearly established. The published results range from values as low as $1^{\mathrm{h}} 40^{\mathrm{m}}$ (Szkody \& Feinswog 1988, from a single sine solution of J photometry) to values as high as $3^{\mathrm{h}} 29^{\mathrm{m}}$ (Vogt et al. 1989, from a preliminary study of spectroscopic data with limited spectral resolution). A more recent and comprehensive photometric study by Schaefer et al. (1992) based on data from 114 nights of observations, together with measurements from the literature, has led to the disappointing conclusion that T Pyx has an incoherent photometric period and that the sinusoidal modulation is not closely tied to the orbital period. By analogy with other stars that have variable photometric periods

* Based on observations made with the International Ultraviolet Explorer and de-archived from the ESA VILSPA Database. (possibly related to the superhump phenomenon), Shaefer et al. (1992) suggested an orbital period of near $1^{\mathrm{h}} 45^{\mathrm{m}}$ from the "best" period for the modulation $\left(1^{\mathrm{h}} 49^{\mathrm{m}}\right)$. The relation between the orbital periods of cataclysmic variables and the spectral types of the secondary stars (Smith and Dhillon 1998) indicates that the secondary star in T Pyx is probably an M 4-5 dwarf.

Already in 1979 ground-based imaging by Duerbeck \& Seitter (1979) detected a shell of ejected matter, a few arcsec across, that surrounded the system. Subsequent observations by Seitter (1986) and Duerbeck (1987) revealed the presence of structures in the shell, while Shara et al. (1989) discovered the additional presence of a fainter extended shell with a radius of about 10 arcsec. Thanks to the high spatial resolution of the WFPC2 on board the HST, the "shell" has been resolved into more than two thousand individual knots (Shara et al. 1997).

These observations have provided direct evidence that the ejecta of novae, previously believed to be reasonably homogeneous, are instead highly structured, confirming the GHRS observations by Shore et al. (1993) that established the presence of knots in Nova Cyg 1992 and the indirect conjectures on the existence of knots previously made by Krautter et al. (1984), Williams et al. (1991), and Saizar \& Ferland (1994).

Shabbaz et al. (1997) interpreted the presence of two spectral features on either side of $\mathrm{H} \alpha$ as the signature of a bi-polar, highvelocity, highly collimated jet, but subsequent studies by Margon \& Deutsch (1998) and by O'Brien \& Cohen (1998) have shown 


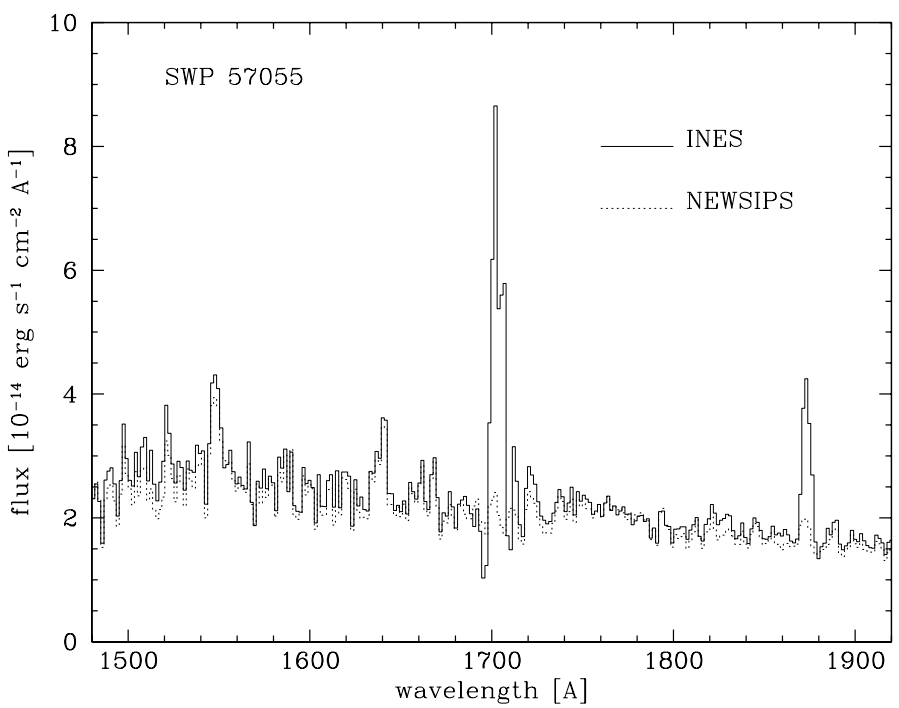

Fig. 1. Comparison of NEWSIPS and INES extraction methods. Note the two strong lines near 1703 and $1873 \AA$ present only in the INES extraction.

that these features are instead emission in the [NII] 6548 and 6584 lines from a complex velocity field in the surrounding shell.

\subsection{The missing outburst}

Observations of an RN for which an outburst is expected to occur provide a unique opportunity for a direct observational test of the theoretical expectations of the thermonuclear runaway (TNR) theory. >From the observed $\dot{M}$ and the inter-outburst interval, one can indeed estimate the total accreted mass $M_{\text {accr }}$ and compare it to both the theoretical mass for ignition $M_{\text {ign }}$ and the observed mass of the ejected shell $M_{\mathrm{ej}}$. Ultraviolet observations are best-suited for these studies since most of the accretion disk luminosity is emitted in the far-UV and many emission lines of important astrophysical species are present in this wavelength range.

With this rationale in mind and in view of the allegedly imminent outburst of T Pyx, as expected on the basis of its apparently regular behavior, with a mean interval of about 19 years between the observed outbursts (WLTO), and of the about twenty years elapsed from the last outburst of December 1966, we started an observing program in 1986 with IUE with the purpose of monitoring the quiescent phase and to follow the spectroscopic changes in the phases prior to the outburst and during the early outburst. Actually, the star has successfully managed to postpone the long-awaited outburst, and at the present time (2006) has surpassed by sixteen years the longest inter-outburst interval so far recorded ( $24 \mathrm{yr})$.

Shortly before this paper neared completion, Schaefer (2005) published the results of a study on the interoutburst interval in recurrent novae. $>$ From an analysis of the data on the magnitudes obtained from archival plates, from the literature, and from his own collection of CCD magnitudes, he found that the product of the inter-eruption interval times the average bolometrically corrected flux is a constant for both T Pyx and U Sco. This finding, together with the apparent decline in the observed B magnitude in the last inter-eruption intervals (Fig. 1 in Shaefer's paper), has provided a physical basis for predicting that the next outburst of T Pyx will occur around 2052.
While waiting to (hopefully) personally verify these predictions, we present and discuss here (Paper I) the main spectroscopic results from 16 years of IUE observations. In a follow-up paper (Selvelli \& Gilmozzi 2006, Paper II), we will combine the results of these observations with other basic information on the system (including the implications of the findings by Schaefer 2005, on the accretion rate of T Pyx during the past inter-eruption intervals), in order to set stringent constraints on the physical parameters of this elusive object and to refine our understanding of the recurrent nova phenomenon.

\section{The IUE observations and data reduction}

The only IUE observations of T Pyx previous to ours were those of May 11, 1980 by Seitter and Duerbeck and reported by Bruch et al. (1981). In this preliminary study they found $E_{B-V} \sim 0.35$ and gave an estimate for the distance of about $2500 \mathrm{pc}$.

The IUE spectra cover the time interval from May 11, 1980 to May 7, 1996 (with a gap between 1980 and 1987). In total, 37 short wavelength (SWP camera) and 14 long wavelength (LWP camera) spectra are available, although some SWP spectra present a low $S / N$ because of high background noise and/or a bad photometric response as a consequence of unusual centering in the IUE aperture. Typical exposure times for the SWP spectra were around three hours with a few exposures twice as long (Table 1).

An important aspect of data reduction is that the IUE data extraction and calibration methods have undergone several revisions during and after the IUE lifetime, which has led to a general improvement in the quality of the line spectrum. While this work was in progress (from 1987 to the last spectra secured in May 1996), both the IUESIPS and then NEWSIPS extraction procedures were adopted. See Gilmozzi et al. (1998) for some considerations of the difference in the quality of data extraction using IUESIPS or NEWSIPS.

The spectra used in this paper were instead retrieved from the INES (IUE Newly Extracted Spectra) final archive. The more relevant modifications in the INES system, in comparison with NEWSIPS, are: 1) the adoption of a new noise model; 2) a more accurate representation of the spatial profile of the spectrum; 3) a more reliable determination of the background; 4) a more adequate treatment of "bad" pixels; and 5) the improvement in the handling and propagation of the quality flags to the final extracted spectra. In general, the extracted fluxes of INES and NEWSIPS are in excellent agreement, but especially for spectra with weak continua and narrow emission lines the INES extraction can register significantly more flux (up to ten sigma!) (Schartel and Skillen 1998). For a detailed description of the IUE-INES system see Rodriguez-Pascual et al. (1999) and Gonzalez-Riestra et al. (2001).

For T Pyx, a comparison between the NEWSIPS and INES extraction methods of the same spectrum has shown in several cases remarkable differences in the strength of some emission features. Figure 1 (SWP57055) is an outstanding example of this problem that may surely help to emphasize the difficulties we have had in assessing the reality of some spectral features. The features at 1703 and $1873 \AA$ in the INES extracted spectrum are due to knots of emission located outside the strip that defines the spectrum (they are flagged as "cosmic rays" but that they are quite wide and both lie at exactly the same distance from the spectrum might indicate that they may be real spectral features originating from an extended region, see also Fig. 6). While NEWSIPS automatically removes these lines (with the 
Table 1. IUE observation log.

\begin{tabular}{|c|c|c|c|c|}
\hline Camera & Image & Date & ExpTime [s] & Comments \\
\hline LWR & 07724 & 1980-05-11 & 7199.8 & \\
\hline SWP & 08973 & $1980-05-11$ & 12899.8 & \\
\hline LWP & 09204 & 1986-09-27 & 7199.8 & \\
\hline SWP & 29318 & $1986-09-27$ & 16199.5 & \\
\hline SWP & 32218 & 1987-11-02 & 16799.6 & \\
\hline LWP & 11996 & $1987-11-02$ & 6599.7 & \\
\hline LWP & 12644 & 1988-02-11 & 7199.8 & \\
\hline SWP & 32899 & $1988-02-11$ & 12779.8 & \\
\hline LWP & 12791 & 1988-03-03 & 12779.8 & \\
\hline SWP & 33034 & 1988-03-04 & 23579.7 & \\
\hline LWP & 14383 & 1988-11-05 & 7799.4 & \\
\hline SWP & 34696 & $1988-11-05$ & 17159.6 & \\
\hline LWP & 16757 & 1989-11-07 & 7799.4 & \\
\hline SWP & 37536 & $1989-11-07$ & 16799.6 & \\
\hline SWP & 43442 & $1991-12-22$ & 15599.4 & \\
\hline LWP & 22052 & $1991-12-22$ & 7199.8 & \\
\hline SWP & 44182 & 1992-03-16 & 14999.8 & \\
\hline LWP & 22608 & 1992-03-16 & 9599.6 & \\
\hline SWP & 44948 & $1992-06-17$ & 16799.6 & \\
\hline LWP & 23317 & $1992-06-18$ & 6899.5 & \\
\hline LWP & 24612 & $1992-12-28$ & 8399.5 & \\
\hline SWP & 46605 & $1992-12-28$ & 16319.5 & \\
\hline SWP & 47057 & $1993-02-27$ & 16499.7 & \\
\hline LWP & 25020 & $1993-02-27$ & 6599.7 & \\
\hline SWP & 47323 & $1993-03-20$ & 20099.7 & poor center \\
\hline SWP & 47328 & 1993-03-21 & 17999.7 & poor center \\
\hline SWP & 47332 & $1993-03-22$ & 23099.6 & \\
\hline SWP & 49365 & $1993-11-29$ & 9599.6 & noisy \\
\hline SWP & 49366 & $1993-11-29$ & 10679.7 & noisy \\
\hline SWP & 50099 & 1994-02-24 & 11399.8 & \\
\hline SWP & 50100 & 1994-02-24 & 10799.7 & noisy \\
\hline SWP & 50596 & 1994-04-20 & 24479.6 & \\
\hline SWP & 52886 & $1994-11-23$ & 8999.6 & \\
\hline SWP & 52887 & $1994-11-23$ & 9479.6 & noisy \\
\hline SWP & 53809 & $1995-02-02$ & 11399.8 & \\
\hline SWP & 53810 & $1995-02-02$ & 10679.7 & noisy \\
\hline SWP & 54590 & $1995-05-03$ & 11099.6 & \\
\hline SWP & 54591 & $1995-05-04$ & 11699.6 & noisy \\
\hline SWP & 56240 & $1995-11-26$ & 11999.5 & \\
\hline SWP & 57030 & $1996-05-01$ & 11999.5 & \\
\hline SWP & 57031 & $1996-05-02$ & 12599.5 & \\
\hline SWP & 57032 & $1996-05-02$ & 11999.5 & \\
\hline SWP & 57033 & $1996-05-03$ & 11999.5 & \\
\hline SWP & 57034 & $1996-05-03$ & 10799.7 & \\
\hline SWP & 57035 & $1996-05-03$ & 6599.7 & \\
\hline SWP & 57039 & $1996-05-04$ & 7799.4 & \\
\hline SWP & 57042 & $1996-05-04$ & 9899.4 & \\
\hline SWP & 57047 & $1996-05-05$ & 10799.7 & \\
\hline LWP & 32286 & $1996-05-05$ & 5699.8 & \\
\hline SWP & 57055 & $1996-05-06$ & 10799.7 & \\
\hline LWP & 32287 & $1996-05-06$ & 6599.7 & \\
\hline
\end{tabular}

risk of removing also real emission features), INES is more conservative and keeps the features but puts a proper quality flag in the corresponding spectrum column. In view of these problems that are especially critical when one deals with the spectrum of an object that is surrounded by an extended shell, we have carefully checked all line-by-line (SILO) images for the presence of similar pseudo-emission features in order to guarantee the reality of the emission features that are present in the various INES extracted spectra (see also Sect. 4.2).

Another non minor consequence of the migration from IUESIPS to NEWSIPS and finally to INES is in the effects that the changes in the extraction methods and in the absolute

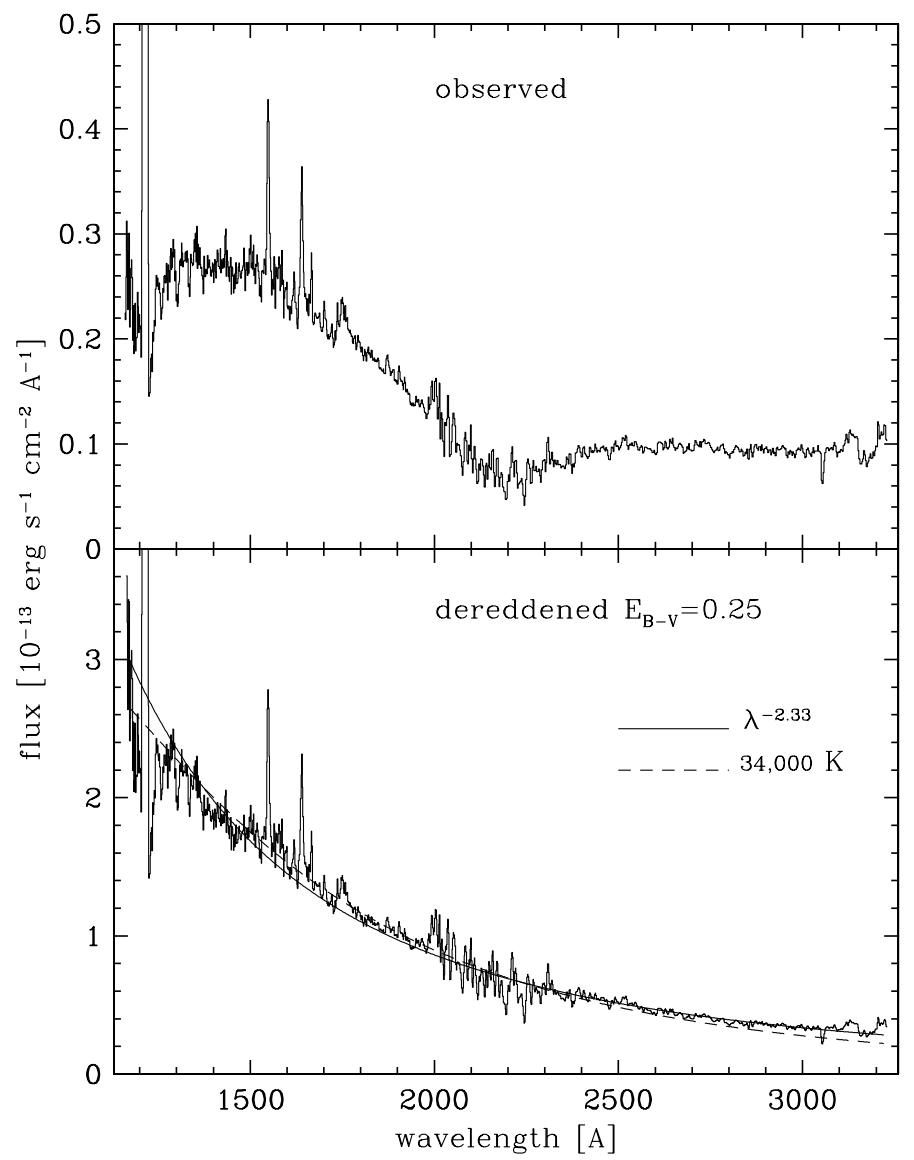

Fig. 2. Average IUE spectrum of T Pyx obtained by co-adding and merging $35 \mathrm{SW}$ and $14 \mathrm{LW}$ IUE spectra. Top: observed, bottom: dereddened with $E_{B-V}=0.25$ (this paper).

calibration tables have had on the shape of the continuum and consequently on the depth of the interstellar absorption bump. In turn, this has resulted in non negligible changes in our estimates of the reddening and of the UV-integrated flux and consequently on the distance, the UV luminosity, and ultimately the mass accretion rate (see Paper II).

\section{The continuum energy distribution}

The observed UV continuum distribution of T Pyx has remained nearly constant in slope and intensity over the 16 years of IUE observations showing only minor variations and no indication of any long term trends. In the SWP region the standard deviation is only $3.7 \%$, even including the badly exposed spectra. This has justified the creation of an average spectrum (Fig. 2, top) by co-adding and merging all SWP and LW spectra that are not underexposed or badly centered.

The improved $S / N$ in the average spectrum has allowed both the detection of weak line features and an accurate determination of the reddening. We obtained $E_{B-V}=0.25 \pm 0.02$ by the standard method of removing the broad interstellar extinction feature centered at $2175 \AA$ using the average interstellar absorption curve of Savage and Mathis (1978). Therefore in the present study and in Paper II we adopt $A_{\mathrm{v}}=3.15 \times 0.25 \sim 0.78$. The same method applied earlier to the smaller sample of spectra processed with IUESIPS gave $E_{B-V}=0.31$, while for NEWSIPS spectra it gave $E_{B-V}=0.24$.

One should note that there is some confusion in the literature about the $E_{B-V}$ value for T Pyx: WLTO used the preliminary 
value $E_{B-V}=0.35$ by Bruch et al. (1981, but they reported it as $0.36)$ and therefore obtained dereddened colors $(B-V)_{\mathrm{o}}=-0.26$ and $(U-B)_{\mathrm{o}}=-1.25$, which look too negative. These same colors were subsequently adopted by Patterson et al. (1998), who on this basis concluded that "T Pyx is the bluest nova remnant in the sky". Bruch and Engel (1994) reported $E_{B-V}=0.20$ and $(B-V)=0.14$ from which they derived $(B-V)_{\mathrm{o}}=-0.06$. (This $E_{B-V}=0.20$ value is probably a typo, because they give Bruch et al. 1981, as a reference, but, unfortunately, this same value was listed also by Szkody 1994). Finally, Weight et al. (1994) gave $E_{B-V}=0.08$ in a near-IR study of old classical novae.

The UV continuum is clearly defined longwards of $1400 \AA$ where only a few and well-known emission lines are present in the SWP spectra, while the LW region is poorer in line features. For a correct positioning of the continuum in the region below $1400 \AA$, where it was not clear whether the spectral features were actually in absorption or in emission, we have been helped by a preliminary identification of these line features (most of them come out as absorption lines, see Sect. 5). The presence of a wide interstellar or circumstellar Ly $\alpha$ absorption, which extends longward up to $1250 \AA$, together with that of the strong Ly $\alpha$ emission of geocoronal origin has also made it rather difficult to estimate the continuum below $1260 \AA$. Therefore, we first determined the continuum in the range 1260-3200 $\AA$ using only line-free regions, fitted it with a spline, and extrapolated it to shorter wavelengths. A less interactive fitting method (the icfit task in IRAF) yielded a very similar result.

The best single-curve fit to the UV continuum is a powerlaw distribution $F_{\lambda}=4.2810^{-6} \lambda^{-2.331}\left(\mathrm{erg} \mathrm{cm}^{-2} \mathrm{~s}^{-1} \AA^{-1}\right)$, with a small uncertainty of \pm 0.04 in the index. This spectral index is exactly the Lynden-Bell law for a standard disk $(\alpha=-2.33)$. A black body with $T=34160 \mathrm{~K}$ is also a good fit, although it would be difficult to ascribe a single temperature to the most plausible source of the continuum, the accretion disk. The lower half of Fig. 2 shows a plot of the dereddened average spectrum together with the continuum fits (see also Fig. 8). The $\lambda \lambda 1180$ 3230 integrated flux of both the reddening-corrected average continuum and the power-law fit is $1.94 \times 10^{-10} \mathrm{erg} \mathrm{cm}^{-2} \mathrm{~s}^{-1}$.

\section{The emission line spectrum}

The strongest emission features are CIV 1550 and HeII 1640 (Fig. 3, top), while weaker emission lines are identified as OIII] 1666, NIII] 1750, etc. (see Table 2 for a list of additional emission lines in the average spectrum, together with their tentative identification and their reddening-corrected emission intensity). The LW region seems almost featureless, but a closer inspection reveals marginal evidence of weak emission and absorption features. The emission line near $3135 \AA$ is identified as OIII 3134 , a Bowen fluorescence line excited by the HeII Ly- $\alpha$ transition at $303.8 \AA$.

An examination of the individual spectra shows that only the emission lines of CIV 1550 and HeII 1640 are present in all spectra, although quite variable, while OIII] 1666 and NIII] 1750 are present in about one half of the spectra. The changes in intensity of the emission lines of CIV 1550 and HeII 1640 are associated to changes in the line width. Table 3 gives the observed equivalent width $(E W)$ and $F W H M$ for these emission lines, although the limited spectral resolution of the data has precluded very accurate measurements. A good correlation (rms of residuals $=1.015$ ) exists between the $F W H M$ and the $E W$ of the HeII 1640 line (Fig. 4). The corresponding correlation for the CIV 1550 emission line is poorer (rms of residuals $=1.579$ ),

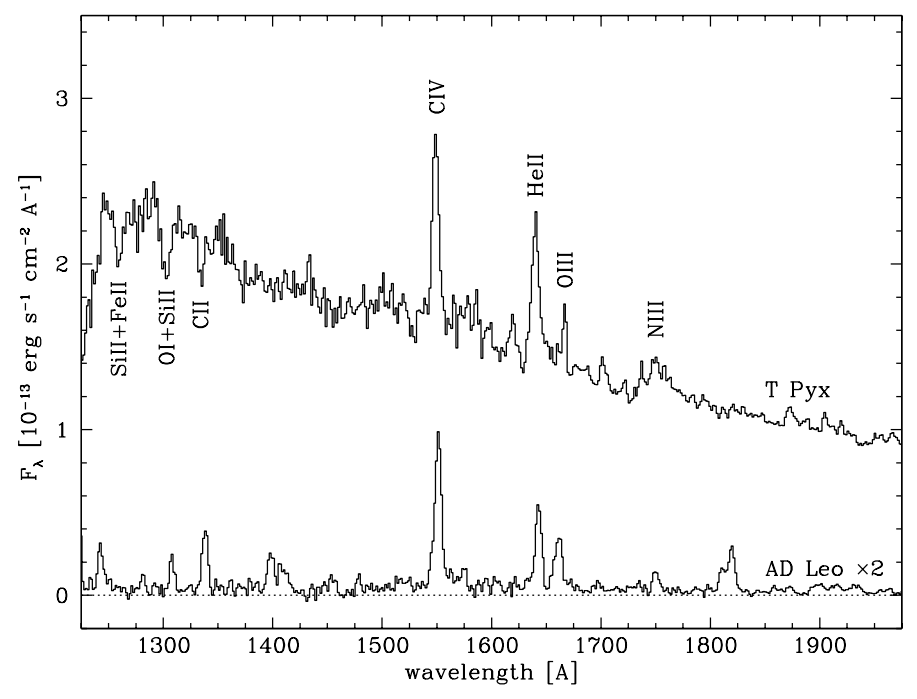

Fig. 3. Details of the dereddened average spectrum of T Pyx in the SW range. The spectrum of the late-type active star AD-Leo (bottom) is included for comparison (see Sect. 6.2).

Table 2. The intensities of the emission lines in the average spectrum and their proposed identifications.

\begin{tabular}{|c|c|c|}
\hline $\begin{array}{l}\text { Wavelength }^{a} \\
(\AA)\end{array}$ & $\begin{array}{c}\text { Intensity } \\
\left(10^{-13} \mathrm{erg} \mathrm{cm}^{-2} \mathrm{~s}^{-1}\right)\end{array}$ & Identification $^{b}$ \\
\hline 1177 & 1.98 & CIII(4) 75.70 \\
\hline 1291 & 1.02 & $\mathrm{CI}(4.01) 87.82 ?$ \\
\hline 1354 & 0.50 & $\begin{array}{l}\mathrm{OI}(1) 55.60+\mathrm{CI}(43) 54.28+ \\
\mathrm{CI}(42) 55.84\end{array}$ \\
\hline 1433 & 0.62 & unidentified (also in HR Del) \\
\hline 1502 & 0.73 & SiIII 1501 (diel.) ? \\
\hline 1510 & 0.41 & OV(64) $06.76 ?$ \\
\hline 1520 & 0.37 & unidentified \\
\hline 1550 & 8.35 & CIV(1) 49.50 \\
\hline 1565 & 0.62 & CI(UV3) $60.86 ?$ \\
\hline 1578 & 0.72 & CIII(UV12.03) $77.25 ?$ \\
\hline 1586 & 0.93 & OIII(19.23) $87.53 ?$ \\
\hline 1620 & 0.72 & $\begin{array}{l}(\mathrm{CIII}(11.72) 20.30+ \\
\mathrm{NV}(53) 19.74) ?\end{array}$ \\
\hline 1640 & 6.32 & HeII(12) 1640.39 \\
\hline 1667 & 1.26 & OIII(0.01) 64.15 \\
\hline 1702 & 0.22 & OV(67) $07.99 ?$ \\
\hline 1738 & 0.30 & NII(13.25) $42.25 ?$ \\
\hline 1750 & 2.32 & NIII(0.01) 50.46 \\
\hline 1872 & 0.63 & unidentified \\
\hline 1905 & 0.35 & CIII(0.01) 06.67 \\
\hline 1920 & 0.16 & CIII(12.02) $23.13 ?$ \\
\hline 2308 & 1.22 & $\operatorname{HeII}(\mathrm{Pa}) 06.20+?$ \\
\hline 2392 & 0.37 & $\mathrm{HeII}(\mathrm{Pa}) 85.40 ?$ \\
\hline 2516 & 0.74 & $\mathrm{HeII}(\mathrm{Pa}) 11.21 ?+?$ \\
\hline 2733 & 0.41 & $\mathrm{HeII}(\mathrm{Pa}) 33.32$ \\
\hline 3134 & 1.84 & OIII(12) 32.79 (Bowen) \\
\hline
\end{tabular}

${ }^{a}$ The listed wavelength is the mean multiplet wavelength; ${ }^{b}$ identifications marked with a (?) are doubtful.

while the correlation between the fluxes in the HeII 1640 and CIV 1550 lines is just fair.

\subsection{The emission lines present in individual spectra only}

One peculiar aspect of the line spectrum of T Pyx is that the emission lines show substantial changes in intensity and detectability in the individual spectra, in sharp contrast to the near 
Table 3. The equivalent widths $(E W)$ and $F W H M$ for the emission lines of CIV 1550 and HeII 1640 in individual spectra.

\begin{tabular}{ccccc}
\hline \hline & \multicolumn{2}{c}{ CIV 1550 } & \multicolumn{2}{c}{ HeII 1640 } \\
SWP & $F W H M$ & $E W$ & $F W H M$ & $E W$ \\
& {$[\AA]$} & {$[\AA]$} & {$[\AA]$} & {$[\AA]$} \\
\hline 08973 & 11.6 & 6.7 & 6.8 & 4.7 \\
29318 & 3.7 & 6.4 & 6.3 & 5.8 \\
32218 & 6.2 & 4.4 & 5.6 & 4.8 \\
32899 & 6.0 & 5.5 & 8.3 & 6.1 \\
33034 & 6.4 & 11.5 & 6.0 & 4.6 \\
34696 & 5.1 & 4.9 & 5.0 & 4.4 \\
37536 & 5.0 & 4.1 & 7.6 & 4.0 \\
43442 & 7.8 & 5.6 & 4.2 & 2.1 \\
44182 & 6.3 & 4.5 & 4.9 & 3.0 \\
44948 & 7.4 & 5.5 & 3.3 & 2.2 \\
46605 & 5.8 & 3.6 & 13.7 & 11.8 \\
47057 & 7.0 & 4.5 & 4.9 & 3.6 \\
47323 & 6.4 & 3.9 & 4.2 & 2.8 \\
47328 & 6.7 & 4.8 & 8.2 & 3.7 \\
47332 & 5.4 & 4.1 & 8.3 & 7.5 \\
49365 & 8.6 & 6.9 & 9.5 & 6.6 \\
49366 & 7.8 & 5.5 & 7.9 & 4.3 \\
50099 & 6.5 & 4.0 & 5.5 & 2.3 \\
50100 & 5. & 3.6 & 8.3 & 7.5 \\
50596 & 6.3 & 4.3 & 7.7 & 5.9 \\
52886 & 4.0 & 4.4 & 5.5 & 2.8 \\
52887 & 4.5 & 4.4 & 5.4 & 2.9 \\
53809 & 6.1 & 4.8 & 8.3 & 6.2 \\
53810 & 7.3 & 5.8 & 5.7 & 2.7 \\
54590 & 10.2 & 4.6 & 9.5 & 4.6 \\
56240 & 8.7 & 6.6 & 4.6 & 1.8 \\
57030 & 6.3 & 6.6 & 5.7 & 3.2 \\
57031 & 5.9 & 5.2 & 8.0 & 4.6 \\
57032 & 6.1 & 5.0 & 5.8 & 3.7 \\
57033 & 6.0 & 6.1 & 6.1 & 3.7 \\
57034 & 6.7 & 6.9 & 5.6 & 2.5 \\
57035 & 7.1 & 8.4 & 7.6 & 6.2 \\
57039 & 5.6 & 4.8 & 3.6 & 0.9 \\
57042 & 4.0 & 4.1 & 5.5 & 2.1 \\
57047 & 3.1 & 2.0 & 8.0 & 6.2 \\
57055 & 5.7 & 4.4 & 4.6 & 2.4 \\
\hline & & & & \\
& & & \\
57.5 &
\end{tabular}

constancy of the continuum (see the SWP spectrum gallery in Fig. 5).

This behavior may cast some doubt on the reality of some emission features, as some of them may be spurious features produced e.g. by cosmic rays hits, due to the long exposure times (more than three hours). A detailed and specific inspection of all the line-by-line (SILO) spectral images has confirmed, however, that several of these features are real emission lines, with only a few being spurious. It is surprising, and somewhat worrisome, that in the extracted spectra of LWP12791 and LWP22052 strong and wide emissions features are present in the region 3100-3250 A but absent in the SILO image. All SILO images show that the strong Ly $\alpha$ emission is of geocoronal origin, as expected on account of the long exposure times.

Table 4 gives the wavelength and the (tentative) identification for the emission feature considered as real. For many of these emissions the identifications are rather uncertain since they are uncommon in other CVs.

\subsection{The features outside the spectrum}

It is generally assumed that the shape of the large entrance aperture of the IUE SWP spectrograph is that of a rectangle whose

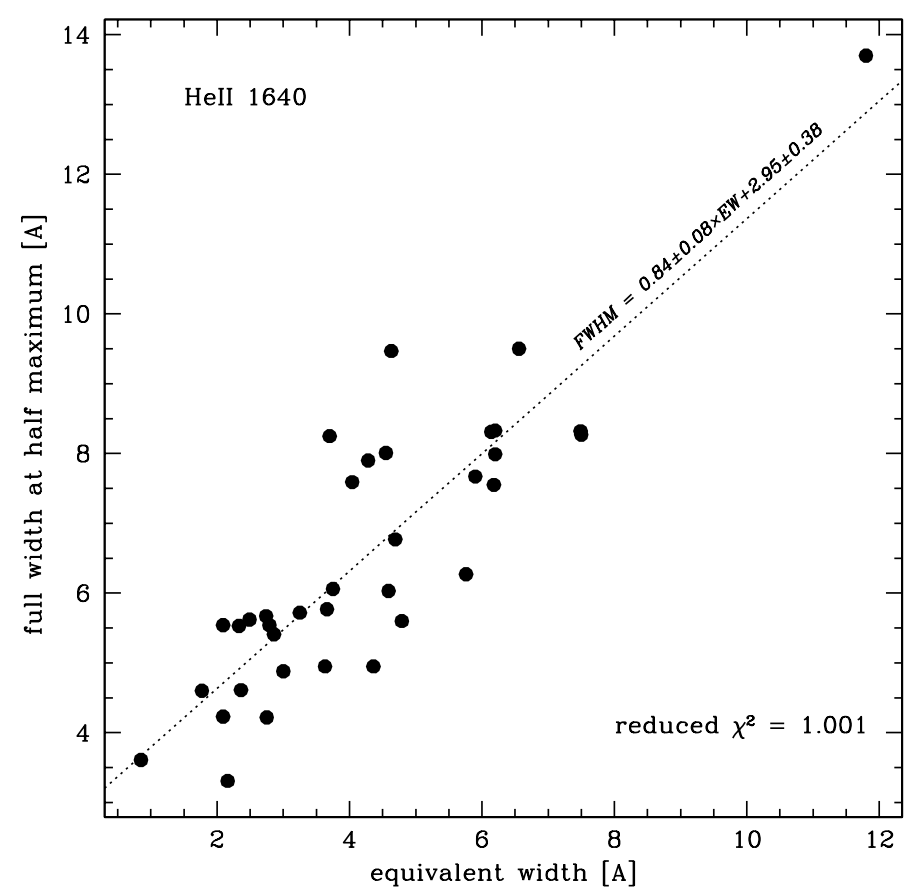

Fig. 4. Correlation between the $F W H M$ and the equivalent width for the HeII 1640 Å emission line.

projected size on the camera faceplate has approximate dimensions of $10 \times 20$ arcsec. The direction of the dispersion is nearly perpendicular to the major axis of the aperture. Therefore, on the SILO images of an extended object (nebula), one expects to see spectral features on both sides of the star spectrum, out to a distance of \pm 10 arcsec.

Actually, the IUE large apertures have parallel sides and rounded ends (Bohlin et al. (1980). The full length to the tips of the rounded ends and the width of the SWP's large aperture were measured before flight and the effective dimensions are $23.0 \times$ 10.3 arcsec. The IUE NEWSIPS manual, Table 2.1 (Garhart et al. 1997) instead gives a large aperture length of $21.65 \pm$ 0.39 arcsec and a large aperture width of $9.07 \pm 0.11$ arcsec, but the reported large aperture area $\left(215.33 \operatorname{arcsec}^{2}\right)$ is quite a bit larger than the product of the quoted length and width, and it is probable that the length does not include the two rounded ends. The precise form and size of the IUE large aperture is apparently not well-defined (see also Gonzalez-Delgado \& Perez 2001), but can be considered as intermediate between a rectangle and an ellipse. In the present study we conservatively chose to consider only features at a distance of $\leq 10$ arcsec on either side of the central strip for well-centered objects. The only notable exception is SWP47332 (see below).

The inspection of all SILO images led to the detection of several emission spikes outside the central strip of the spectrum but still inside the 20 arcsec large aperture. Some pseudo-emission features are produced by the permanent presence of "hot pixels" (for example, a typical one falls near $1750 \AA$, nearly the same wavelength as a well-known NIII] line, though outside the typical position of well-centered spectra). It is also possible that several of these spikes are caused by cosmic ray hits mimicking the PSF of IUE. However, there are cases in which the spikes either are strictly associated with known emission features present in the (main) spectrum or show a common pattern hardly attributable to the impact of cosmic rays.

For example: in SWP49365 a spike at $1243 \AA$ is at the same wavelength as a spectral feature, probably NV 1240; in 


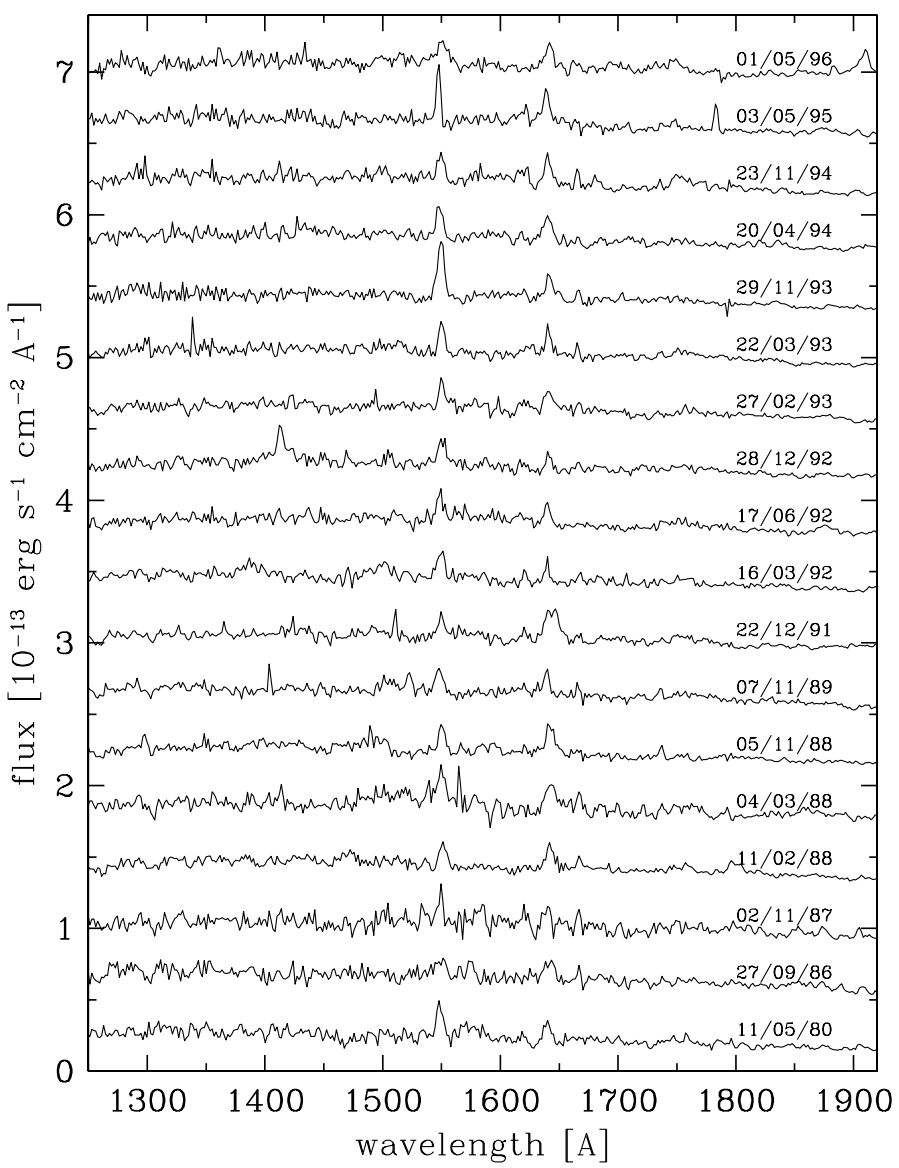

Fig. 5. SWP observed spectra gallery. Spectra are offset by $4 \times$ $10^{-14} \mathrm{erg} \mathrm{s}^{-1} \mathrm{~cm}^{-2} \mathrm{~A}^{-1}$. The near constancy of the continuum contrasts with the changes in the emission lines.

Table 4. Emission lines present only in a few spectra and not listed in Table 2.

\begin{tabular}{cll}
\hline \hline Wavelength $^{a}$ & Identification $^{b}$ & SWP spectrum \\
\hline 1190 & SIII(1) 90.20 & $29318,37536,44182$, \\
& & 53810,56240 \\
1243 & NV(1) 40.15 & 49365 \\
1250 & CIII(9) 47.38 & 57039 \\
1285 & CI(5) 80.62 & 56240 \\
1371 & OV(7) 71.29 & 08973 \\
1412 & NI(10) 11.97 & 43442 \\
1474 & SI(3,4) 73.77 & 50596 \\
1713 & SiII(10) 11.0 & 57034 \\
1727 & $?$ & 53810 \\
1775 & $?$ & 57039 \\
1782 & FeII(191) 85.27 & 29318, \\
1890 & SiIII(1) 92.03 & 50099 \\
1910 & CIII(0.01) 08.73 & 08973
\end{tabular}

${ }^{a}$ The listed wavelength is the mean multiplet wavelength; ${ }^{b}$ most identifications are tentative.

SWP43442 a knot at 1414 is at nearly the same wavelength as a spectral feature at $1412 \AA$; in SWP33034, SWP53809, and SWP47057, there are spikes near $1545 \AA$ that seem associated with CIV 1550; in SWP57055 there are two strong spikes at 1703 and $1873 \AA$, both lying on the same side of the spectrum, at the same distance (about 9 arcsec) from it (see Fig. 6 right; as already mentioned in Sect. 2 they are present as strong emission lines in the INES extracted spectrum and flagged as "cosmic rays"); the same in SWP54590 at 1751 and $1934 \AA$; finally in SWP08973 a spike at $1909 \AA$ is associated with an emission feature at $1910 \AA$ on the spectrum (probably CIII] 1909).

The case of SWP47332 is instead quite problematic because there are apparently four spikes near $1640 \AA$ (Fig. 6 left) and also four ones near $1400 \AA$ (not in the same pattern, although one is the same distance from the spectrum at both wavelengths). The two stronger spikes on both sides of HeII 1640 would fall outside the large aperture if one assumes the "standard" 20 arcsec size, but they would fall just near the border if one adopts the 23.0 arcsec size given by Bohlin et al. (1980). A comparison with the spatial size (perpendicular to the dispersion) of the geocoronal Ly-alpha (that fills the large aperture) shows that the separation between the two external features near $\lambda 1640$ is close to the spatial width of the geocoronal Ly-alpha, although the saturation effects in the geocoronal line, together with the difference in the spatial point spread function for the two wavelength regions (Cassatella et al. 1985; Garhart et al. 1997, makes it difficult to make a definite statement about the true nature of the two external features. This particular example highlights the difficulties one encounters in establishing the reality of the "external" features in IUE spectra, especially when nature also maliciously conspires to place two symmetrical knots on either side of a wellknown spectral line.

In general, if the external spikes correspond to spectral features, they may originate in the extended region (nebula) surrounding the system, although that only one or, at most, a few external features (and not an entire spectrum of several emission lines) are present in an image is of difficult to explain physically. On the other hand, an examination of the SILOs of other faint emission line objects (Seyfert Galaxies), made for comparison, has not revealed as many spikes features as in T Pyx, although the spectra were taken with similar exposure times.

An analysis of the distribution of spikes inside and outside the large aperture has not been conclusive in demonstrating that the distribution inside is statistically larger than that outside. Apart from small numbers, the probable reason for this is that the background varies substantially in different regions of the image, and even small variations in the threshold (in terms of $\sigma_{\mathrm{bgd}}$ ) for automatic spike detection give different results. If one only uses "bright" spikes, the number within the aperture is often larger than that expected from the average in the frame; but this is difficult to quantify in a totally objective way since the "brightness" of the spikes also depends on the position in the frame. Even so, the result for bright spikes, together with the comparison with similarly exposed spectra, would appear a strong argument in favor of at least some of the spikes being real line emissions.

There are however, no obvious, known candidates that would produce spikes near the edge of the aperture. Figure 7 shows the IUE aperture during the observations in Fig. 6 superimposed on the HST image of T Pyx and its surroundings (Shara et al., 1997). The aperture is plotted with a standard $10^{\prime \prime} \times 20^{\prime \prime}$ size, although the long axis is probably $15 \%$ longer, as explained above. The lack of prominent knots in the shell near the rounded edge of the aperture is not in itself sufficient for excluding the reality of the spikes: the HST image is a composite of observations taken in 1994-95, while the IUE observations were taken in 1993 and 1996 and we know that the spikes are short-term transients, e.g. the ones in SWP 57055 were not present a day before in SWP 57047 (although this point may be considered an argument against the interpretation that they are emission lines). The absence of candidates certainly weakens the interpretation that the spikes are emission lines rather than cosmic rays in the 

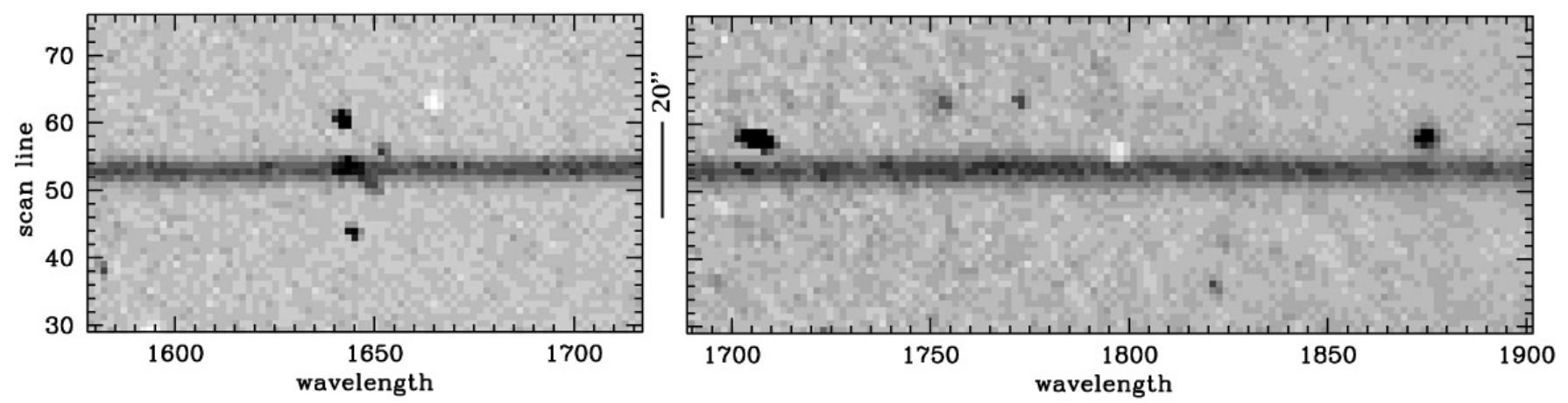

Fig. 6. Line-by-line images of SWP47332 (left) and SWP 57055 (right). The two symmetrical knots on either side of the HeII 1640 line in the left image fall very near the border of the large aperture. Their reality is uncertain, see the text for details. The bright knots just above the spectrum in the image on the right are those responsible for the emission lines at 1703 and $1873 \AA$ in Fig. 1 .

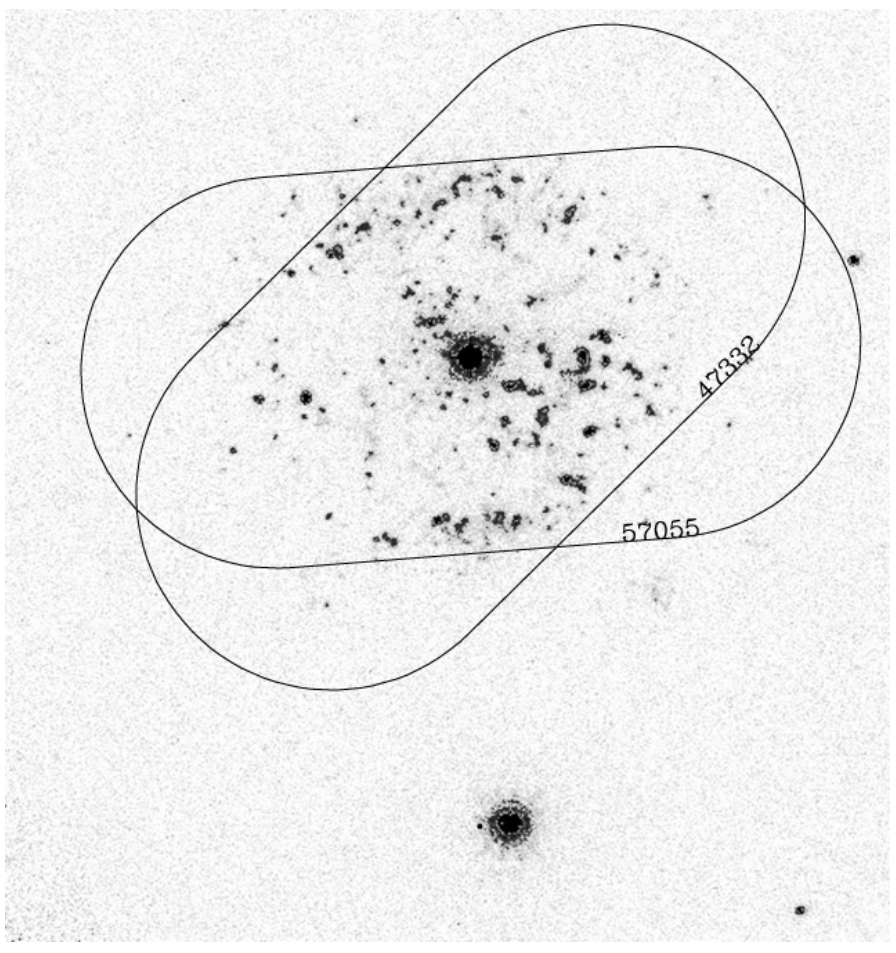

Fig. 7. IUE slit position during the observations reported in Fig. 6 superimposed on an HST image of T Pyx and its "shell". The IUE aperture is drawn with a size of $10^{\prime \prime} \times 20^{\prime \prime}$ (tip to tip), although the long axis is probably $15 \%$ longer. No obviously bright candidates for the transient features detected in 1993 and 1996 by IUE are evident near the edge of the aperture in the composite HST image taken in 1994-1995.

case of SWP 47332. There are instead enough knots at 5-6" from T Pyx that an origin in the nebula for the spikes in SWP 57055 cannot be ruled out.

\section{The absorption lines and the model spectra}

In spite of the limited spectral resolution, the $\operatorname{good} S / N$ in the average spectrum has allowed positive detection of several absorption features (Fig. 3, top). They are all listed in Table 5, together with their corresponding equivalent widths and the identifications. Most of them are identified as well-known resonance lines of once ionized species.

An interstellar contribution to the zero-volt component in some of these lines is expected, but the presence of intrinsic variations in individual spectra (especially for the SiII 1260 line) and
Table 5. Equivalent widths and identifications for the absorption lines in the average spectrum.

\begin{tabular}{|c|c|c|}
\hline Wavelength & $E W[\AA]$ & Identification \\
\hline 1191 & 0.89 & SiII(5) $90.41+$ SIII(1) 90.20 \\
\hline 1259 & 1.15 & $\begin{array}{l}\text { SiII(4) } 60.42+\text { FeII(9) } 60.54 \\
+ \text { SII(1) } 59.52\end{array}$ \\
\hline 1303 & 1.01 & OI(2) 02.17 + SiII(3) 04.37 \\
\hline 1334 & 0.73 & CII(1) 35.10 \\
\hline 1371 & 0.40 & $\operatorname{NiII(8)~} 70.20$ \\
\hline 1529 & 0.52 & $\operatorname{SiII}(2) 26.71$ \\
\hline 1628 & 0.49 & $?$ \\
\hline 1727 & 0.82 & $?$ \\
\hline 1807 & 0.50 & SiII(1) 08.01 \\
\hline 1816 & 0.14 & SiII(1) 16.92 \\
\hline 2376 & 1.35 & FeII(2) 73.73 \\
\hline 2475 & 1.00 & $\operatorname{TiII}(2) 78.64$ \\
\hline 2600 & 0.75 & Fe II(1) $99.39+\mathrm{MnII}(1) 05.70$ \\
\hline 2799 & 1.06 & $\operatorname{MgII}(1) 97.92+$ \\
\hline 2853 & 0.41 & $\operatorname{MgI}(1) 52.12$ \\
\hline
\end{tabular}

the weakness of the absorption near MgII 2800 indicate that it is not dominant. Unfortunately, the limited resolution of the IUE low resolution spectra has not allowed the intrinsic profile of the lines to be appreciated and the probable presence of more than one contributor to be detected.

It is probable that most of the absorption lines originate in the accretion disk itself. Wade \& Hubeny (1998) present a large grid of computed spectra from steady-state accretion disks in luminous CVs. Disk spectra corresponding to twenty-six different combinations of accretion rates and WD masses are computed and tabulated for six different disk inclination angles $i$. The wavelength coverage of the models ranges from 800 to $2000 \AA$ so it is possible to compare them with the IUE spectra taken with the SWP camera (1160-1960 ̊). The spectral features we used for the fitting are the intensity and width of the undisplaced absorption lines (e.g. SiII 1260, OI + SiII 1305, CII 1335, etc). Most of the 156 different models were compared with the reddening-corrected average SWP spectrum of T Pyx but, disappointingly, while there is quite a range of values that gives a satisfactory agreement with the observed depth and shape of the absorption lines, no single solution is convincingly valid at the same time both for the continuum distribution and the depth and shape of the absorption lines. The best fits for the shape and depth of the lines (models $e e$ and $j j$ at rather low inclination angles) correspond to high values for the white-dwarf mass 
$\left(M_{1} \sim 1.21 M_{\odot}\right.$ and $\left.\sim 1.03 M_{\odot}\right)$, but give a continuum that is too steep.

\section{Discussion}

In the following three subsections we will first compare the UV spectrum of T Pyx with that of other ex-novae, then we will suggests some mechanisms to explain the origin of the UV emission lines and their variations, and finally we will consider the origin of the observed optical and IR magnitudes of T Pyx.

\subsection{T Pyx and other ex-novae in the UV}

We compared the dereddened UV spectrum of T Pyx with all the available IUE spectra of novae in quiescence (about 18 objects, including V603 Aql, RR Pic, HR Del, V533 Her, V841 Oph, etc.).

Surprisingly, the best agreement for the shape of the continuum and the intensity of the emission lines has been found with the very-slow nova HR Del (see also Selvelli \& Friedjung, 2003), whose UV spectrum would be almost indistinguishable from that of T Pyx, were it not for the two absorption components in the NV 1240 and CIV 1550 resonance lines that are prominent features in HR Del (Fig. 8). This remarkable similarity in the continua of the two stars is quite a challenge to common interpretations: T Pyx is allegedly observed at rather low inclination $(i \sim 15-20$ degrees) and its WD must be very massive (about $1.2-1.4 M_{\odot}$ ) in order to guarantee outbursts with a very short recurrence time (see also Paper II). HR Del, instead, is observed at medium inclination $(i \sim 45$ degrees) and its WD is allegedly on the light side (about $0.6 M_{\odot}$ ) (see Selvelli \& Friedjung 2003, for a discussion on these latter parameters). If we anticipate from Paper II that the accretion rates in the two stars are quite similar, then one would expect a much hotter (steeper) continuum for T Pyx. The fact that T Pyx and HR Del show the same intensity in the HeII 1640 emission line suggests that these (in principle very different) objects also have very similar temperature and continuum distribution in the EUV region, since the $1640 \AA$ line is a recombination line of $\mathrm{He}^{++}$, whose ionization is controlled by the radiation field shortward of $228 \AA$.

Another peculiarity of $\mathrm{T}$ Pyx is its absorption spectrum. Blue-shifted absorptions components in the resonance lines of high ionization species (CIV, SiIV, NV) are generally seen in the UV spectra of luminous (high mass-transfer rate) cataclysmic variables observed at low inclination (e.g. Nova-like, dwarf novae in outburst, the old novae HR Del and V 603 Aql). The absence of any such signature of outflow in T Pyx (which is luminous and observed at low inclination) indicates that at least one basic ingredient for the onset of outflow is missing in this system.

Moreover, the absence in most spectra of T Pyx of both the NV 1240 and the SiIV 1398 resonance lines is also surprising, since these emissions are common in most UV spectra of cataclysmic variables, and, usually, because of their different sensitivity to temperature effects, the absence of one ion is complemented by the presence of the other (while the intermediate temperature ion CIV 1550 always tends to be present). We can tentatively interpret the absence of SiIV as due to a very hot boundary layer whose hard radiation keeps silicon in ionization states higher than $\mathrm{Si}^{+3}$, but in this case it is hard to understand why there is no evidence of the NV 1240 lines either in emission or in absorption.

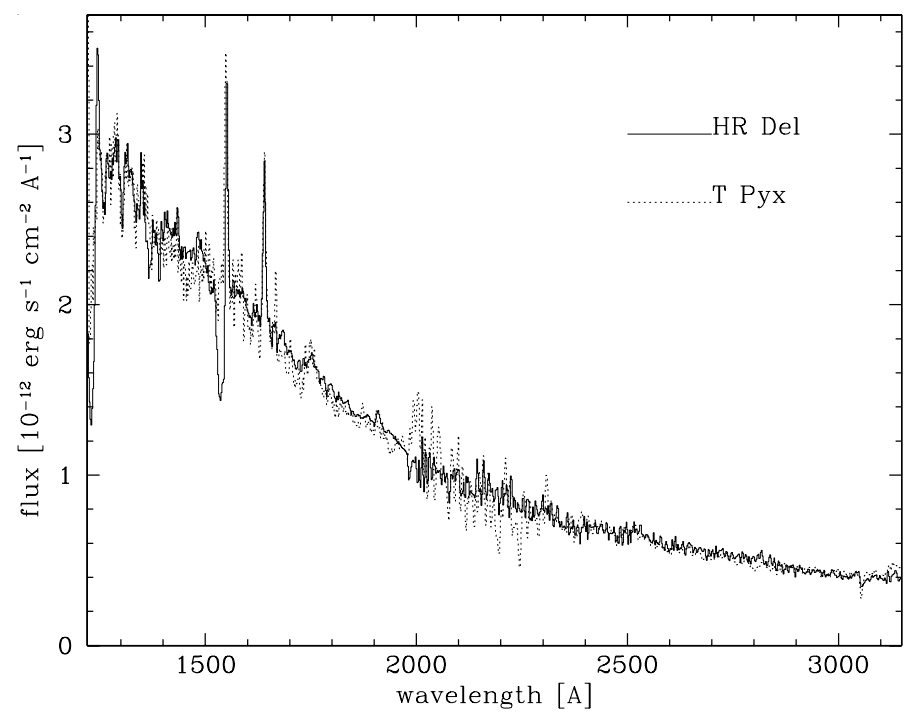

Fig. 8. Comparison between the spectra of T Pyx and HR Del. The two spectra are almost indistinguishable except for the P Cyg component in the CIV 1550 line of HR Del. The spectrum of T Pyx has been scaled to the flux scale of HR Del.

In the old nova V603 Aql, which is observed at almost the same (low) inclination as T Pyx, both NV 1240 (in absorption) and SiIV 1400 (in emission) are present. Also in the old nova RR Pic the line excitation is much higher than in T Pyx: the NV $1240 \AA$, CIV $1550 \AA$, and HeII $1640 \AA$ emission lines are much stronger than in T Pyx. This point will be further discussed in Paper II in the context of the alleged SSS nature of T Pyx, as proposed by Patterson et al. (1998).

Common nebular lines (e.g. NIV] 1483, SiIII] 1892, CIII] 1909 , etc.) are usually absent in the UV spectrum of T Pyx, in contrast to the presence of a (composite) nebular shell surrounding the central object. It is tempting to explain this with the fact that the nebula has a quite small covering factor because of its knot structure and therefore most of the Lyman continuum radiation of the central hot source leaves the star undisturbed and is not degraded into line radiation (HI emissions plus coolants). However, the presence of conspicuous emission lines of HI and HeII casts some doubts on this interpretation. Another possibility is that the near absence of nebular lines could simply be a density effect, the knots' condensations having electron densities higher than the critical ones for the relevant emission lines.

\subsection{Speculations on the origin of the UV emission lines and their variations}

The emission line spectrum of T Pyx shows some peculiarities whose interpretation is not straightforward:

1. the strongest emission lines (CIV 1550, HeII 1640) are variable in intensity and width;

2. some lines are observed in a few spectra only and are uncommon in CVs and/or lack reliable identification;

3. some lines appear inside the slit (not on the spectrum) at uncommon wavelengths and varying positions.

The observed emission-line variations, which in some cases take place on time scales as short as a few hours (see the spectra taken in sequence in May 1996), are not caused by instrumental effects, i.e. different centerings in the IUE aperture that could produce different contributions from the nebular shell. In all the 
SILO images we have examined (with the few exceptions mentioned in Sect. 4.2), there was no evidence of any contribution from an extended region.

The IUE Roll angle (which determines the slit's position angle) is fixed during one exposure, but varies at different epochs. Therefore, knots that may be in the $10 \times 20$ arcsec aperture at one epoch may not be at another, although knots within 10 arcsec of the star are always in the aperture. Even for these, at different position angles the same knot will produce lines that are not only at a different distance from the main spectrum (or even on it) but also at different wavelengths from epoch to epoch (up to $\pm 8 \AA$ depending on the geometry inside the slit). This may help explain the sporadic appearance of lines on and outside the spectrum but, of course, not the variations of the known lines.

Also, the variations can hardly be ascribed to aspectdependent and/or orbital and geometrical effects (bulges, hot spots, etc.) because the system is allegedly viewed nearly poleon $\left(i \leq 20^{\circ}\right.$, Paper II) and the average exposure time of all spectra is longer than the orbital period so that orbit-dependent effects should be averaged over one orbital period or more. The observed variations in the lines of the spectrum must take place in a region whose angular size is on the order (or less) of the PSF of $I U E\left(\leq 2.7^{\prime \prime}\right)$. This region contains the binary system and the innermost nebula (and that part of the nebula that is projected on the compact source).

In other CVs, intrinsic variations in the emission lines are observed in systems that are not stable accretors (e.g., dwarf novae from low to high states, etc.) when they suffer $\dot{M}$ changes in an optically thin accretion regime. This is apparently not the case for T Pyx. The high luminosity of the UV continuum, remarkably constant over 16 years, indicates stable accretion in an optically thick regime.

Besides the difficulty of explaining the variations, the problem also remains with the unidentified or poorly identified features. It is probable that these emission lines may come from regions other than the hot component (accretion disk plus boundary layer), so we tentatively suggest these non mutuallyexclusive regions and/or mechanisms:

\section{1) The (irradiated) chromosphere of the M dwarf companion.}

Several short-period detached binary systems are known e.g.: V471 Tau (Guinan 1990; Young et al. 1988), BE UMa (Ferguson et al. 1991), Feige 24 (Vennes et al. 1991) - which show irradiation induced effects: the atmosphere of the companion star, usually a low-MS star, is heated by the flux of the WD leading to an enhancement of line emission. These systems are thought to be pre-cataclysmic binaries (de Kool \& Ritter 1993); see also Jomaron et al. (1993). In some of these systems, e.g. V471 Tau, the atmosphere of the $\mathrm{K}$ star is inhomogeneous and there is evidence of external plasma around it (Young et al. 1991; Guinan 1990). These atmospheric structures may be related to the cool loops or active region plumes observed in the Sun, but are much more extensive in size and are unstable, with a lifetime of a few days.

We suggest that a similar class of phenomena could also be present in $\mathrm{T}$ Pyx, where transient jets or loops from the cool star, excited by the strong UV radiation of the hot component, may be responsible for the variations and peculiarities of its spectrum. However, while the above-mentioned objects are semi-detached binaries in which the luminosity and temperature of the WD is not particularly high, T Pyx is instead a short-period semi-detached system with high ultraviolet luminosity $\left(L_{\mathrm{UV}} \sim 100 L_{\odot}\right.$, Gilmozzi et al. 1998; and Paper II) and rather high average $T(\sim 34100 \mathrm{~K})$, so that much stronger induced effects are expected. Urban (1988) has already pointed out the importance of irradiation effects on the spectrum of T Pyx.

\section{2) The enhanced activity in the secondary and magnetism.}

It is well-known that most $\mathrm{M}$ dwarfs are characterized by a surface magnetic activity that is manifested by non thermal emission in a hot outer atmosphere (see Hawley 1993, for a review). The magnetic activity increases monotonically toward later spectral types, until at type M 5 and later all dwarfs are $\mathrm{dMe}$ or active and show high-temperature UV emission lines. Rotation also plays an important role in this class of phenomena, therefore, it is possible that binarity with a close companion might increase these effects by tidally-induced rapid rotation, together with irradiation and heating. Flare activity is associated with phenomena like coronal loops and coronal mass ejection that have sizes on the order of the stellar radius and time scales on the order of hours (see the observations of AU Mic with EUVE by Katsova, 1996). Also, if the WD in T Pyx is mildly magnetic, the two stars are probably interconnected by a magnetic field that acts as a guide line for the material streaming from the secondary, and the interaction of the two magnetic fields may heat the plasma between the two stars. A representative spectrum of an active star (AD Leo) is given in Fig. 3 (bottom); the three strongest emission lines are those of CIV $\lambda 1550$, HeII $\lambda 1640$, and OIII $\lambda 1660$, with relative intensities similar to those observed in T Pyx.

In the context of these two models, the absence in the UV spectrum of T Pyx of the MgII 2800 emission line, a wellknown indicator of chromospheric activity, is quite disturbing. We can only invoke the somewhat ad hoc explanation that the MgII emission is suppressed by the corresponding absorption component of the accretion disk and by the interstellar medium.

\section{3) Illumination of (moving) knots of the nebula in front of the} compact object.

Shara et al. (1997) report that a few knots are observed to fade or brighten significantly on a timescale of months and interpreted this in terms of collisions and shocks between successive generations of ejecta. From the comparison of the size of the nebula at different epochs, they derived an upper limit of about only $40 \mathrm{~km} \mathrm{~s}^{-1}$ (but assumed $d=1500 \mathrm{pc}$ ) on the systematic expansion velocity of the knots. Instead, both Margon \& Deutsch (1998) and O'Brien \& Cohen (1998), in a study of the alleged jet components in the $\mathrm{H} \alpha$ line (Shabaz et al. 1997), identified these features as due to the [NII] 6548, 6584 lines and interpreted their wavelength shifts as produced by an expansion velocity of about $500 \mathrm{~km} \mathrm{~s}^{-1}$ along the line of sight through the center of the shell. If some of these "variable knots" happen to lie within the IUE PSF they may also explain the emission line changes and the unusual spectroscopic signatures in the UV spectrum of T Pyx.

To summarize this rather speculative section, we propose that the peculiar spectrum of T Pyx may be due to complex velocity fields in the material (cool loops, active region plumes, transient jets, coronal loops, etc.) that streams from the active companion toward the WD and/or to the transient appearance of high-velocity emitting knots in front of the compact object. That in individual UV spectra only a few extra emission lines (most of them of dubious identification) are observed, instead of a spectrum, means that interpretation is difficult and that quite uncommon physical conditions are required.

The data we have are too sparse to test other possible effects contributing to the unusual behavior of $\mathrm{T}$ Pyx. For 


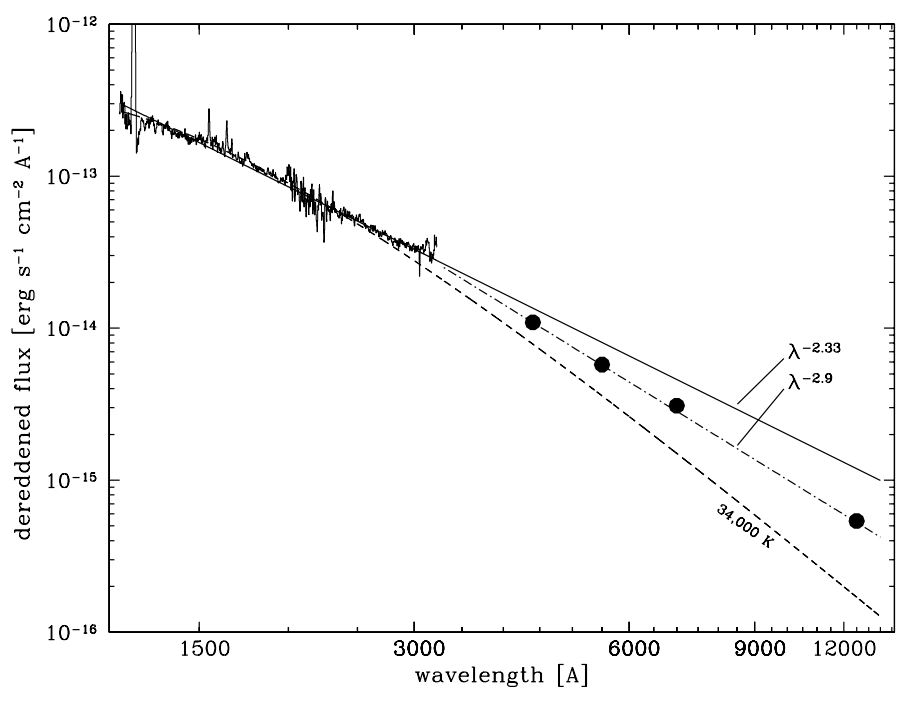

Fig. 9. UV-Opt-IR "spectrum" of T Pyx. The $B, V, R, J$ average fluxes are indicated. The hot source is clearly the main contributor at all wavelengths (the departure from the -2.33 power-law at long wavelengths probably indicates that these fluxes arise in the outer part of the disk, where it becomes optically thin or reaches a physical edge). The $\alpha=-2.9$ power-law is an aid to the eye. The black body that best "fits" the UV continuum (though meaningless for an accretion disk) is also plotted.

example, we have considered precession in the system (partially) hiding and revealing at different epochs a possible hot spot as one explanation for the variability in the emission lines, but much more intensive observations would be necessary to test this hypothesis.

We also considered other more "exotic" scenarios in view of the peculiarities of T Pyx, for instance, that some of the uncommon, sporadic lines may be typical lines that are blue- or red-shifted in a jet. But apart from the occasional, correct wavelength ratio between lines being similar to that of HeII/CIV or $\mathrm{NIII}] / \mathrm{HeII}$ (at a few to several thousand $\mathrm{km} \mathrm{s}^{-1}$ ), there is little evidence to support this hypothesis.

\subsection{The origin of the optical and IR magnitudes}

The extrapolation to the $V$ band of the power-law fit of the UV continuum $F_{\lambda}=4.28 \times 10^{-6} \lambda^{-2.331} \mathrm{erg} \mathrm{cm}^{-2} \mathrm{~s}^{-1} \mathrm{~A}^{-1}$ yields a flux $F_{5500}=8.17 \times 10^{-15} \mathrm{erg} \mathrm{cm}^{-2} \mathrm{~s}^{-1} \mathrm{~A}^{-1}$ or $m_{\mathrm{v}} \sim 14.10$. After proper reddening with $A_{\mathrm{v}}=0.78$, the predicted $V$ magnitude is 14.88. Similar calculations for the $B$ band yield a flux $=1.34 \times$ $10^{-14} \mathrm{erg} \mathrm{cm}^{-2} \mathrm{~s}^{-1} \mathrm{~A}^{-1}$ or $m_{\mathrm{b}} \sim 14.23$. After reddening using $A_{B}=4.1 \times E_{B-V}=1.03$, this becomes $B=15.26$. Therefore, the observed $B=15.5$ (Schaefer (2005) and $V=15.3$ (Downes et al. 1997; Szkody \& Feinswog 1988) magnitudes of T Pyx fall quite close to the values defined by the tail of the power-law fit to the UV continuum. This indicates that $m_{B}$ and $m_{V}$ are dominated by the hot component (accretion disk). That the extrapolated $B-$ $V$ value (after reddening) is close to 0.38 and larger than the observed one (close to 0.20 ) is due to the fact that the spectrum departs from the power-law extrapolation at longer wavelengths (see below and Fig. 9).

The extrapolation to $12500 \AA$ ( $J$ band) gives $1.20 \times$ $10^{-15} \mathrm{erg} \mathrm{cm}^{-2} \mathrm{~s}^{-1} A^{-1}$. After conversion to $J$ magnitudes and reddening with $A_{J}=0.87 \times E_{B-V}=0.22$, we obtain $J=13.52$, to be compared with the observed $J=14.4-14.65$ (Szkody \& Feinswog 1988; Weight 1994) Therefore also in the $J$ band, it is the IR tail of the hot source that is the main contributor to the observed magnitude.

This progressive departure from the power-law distribution is most probably an indication that the largest contribution of the disk to these wavelengths comes from its outer region, where it becomes optically thin (or reaches a physical edge). With a full spectral coverage, it should be possible to determine the temperature of this region. Judging from the optical and infrared colors, this should not be far from $10000 \mathrm{~K}$.

Szkody \& Feinswog (1988) found a sinusoidal modulation in the $J$ light curve of T Pyx from $J=14.34$ to $J=14.50$ with $P=100 \mathrm{~min}$ (single sine solution) or $P=200 \mathrm{~min}$ (double sine solution), which was interpreted as due to ellipsoidal variations in the secondary, that, allegedly, provided a substantial contribution to the IR light. They then applied the technique of ellipsoidal fitting to derive the system inclination (close to 90 degrees) and the mass ratio $q$ (close to 4 ).

This interpretation, however, is compatible with the observations only if the distance to T Pyx is quite low (and they actually suggested a value close to 350 parsecs). In fact, from various relations available in the literature between $M_{J}$ and other stellar parameters such as the mass, the spectral type, etc. (Henry \& Mc Carthy 1990; Henry \& Mc Carthy 1993) or from the observed $J$ values for objects in clusters with known distances (Legget \& Hawkins 1989), it follows that the M 4-M 5 V secondary star in T Pyx (see the Introduction) has an absolute magnitude $M_{J} \sim 7.8$ (or dimmer). This value, together with the observed $J \sim 14.5$, and $A_{J}=0.22$ would imply a distance to T Pyx of only $198 \mathrm{pc}$ (or smaller), not compatible with any value reported in the literature whose lower limit is about 1050 pc (Shara 1997), while a value near $3500 \mathrm{pc}$ is the most plausible (see Paper II). If the largest value is correct, the apparent $J$ magnitude of the secondary star would be about 20.7 , and would thus contribute to less than 1 percent of the observed $J$ flux.

Therefore the IR tail of the hot source is the main contributor to the observed $J=14.4 \mathrm{mag}$. It is possible, however, that heating (irradiation) of the secondary by the accretion disk (see Sect. 6.2) could provide a partial contribution to the $J$ light and be responsible for its modulation. In any case, the presence of a definite modulation in the $J$ curve and the absence of a similar modulation in the optical, where several photometric periods have been reported, are difficult to interpret if, allegedly, the system is seen at the inclination of $\sim 15$ degrees (Warner 1995).

\section{Conclusions}

The constancy of the UV continuum energy distribution over 16 years of IUE observations is quite remarkable in an object that belongs to the CVs class. This behavior contrasts with the observed changes both in intensity and detectability for the UV emission lines. The strongest emission lines (CIV 1550 and HeII 1640) are clearly variable in intensity and width.

The origin of these variations is unclear, so we suggest that the peculiar behavior of T Pyx may be due either to complex velocity fields in the material that streams from the active companion toward the WD (cool loops, active region plumes, transient jets, coronal loops, etc.) and/or to the transient appearance of high-velocity emitting knots from the nebular shell in front of the compact object.

The best single-curve fit to the dereddened $\left(E_{B-V}=0.25 \pm\right.$ 0.02) UV continuum is a power-law distribution $F_{\lambda}=4.28 \times$ $10^{-6} \lambda^{-2.33} \mathrm{erg} \mathrm{cm}^{-2} \mathrm{~s}^{-1} A^{-1}$, with a small uncertainty of \pm 0.04 in the index. The observed $B=15.5, V=15.3$, and $J=14.4$ mag of T Pyx fall quite close to the values defined by the tail of this 
power-law. This clearly indicates that the hot source (accretion disk) is the main contributor at all wavelengths and that the contribution from the secondary star is negligible, also in the IR.

Acknowledgements. We gratefully thank Angelo Cassatella for his participation in the observations and in the preliminary phase of this investigation. We also thank Rosario Gonzalez-Riestra for helpful technical discussions in the early stages of the long gestation period of this article. We also thank the referee for bringing to our attention the recent paper by Schaefer (2005) that appeared just as this paper was being finished.

\section{References}

Bohlin, R. C., Sparks, W. M., Holm, A. V., Savage, B. D., \& Snijders, M. A. J. 1980, A\&A, 85, 1

Bruch, A., Duerbeck, H. W., \& Seitter, W. C. 1981, Mitt. Astr. Ges., 53, 34

Bruch, A., \& Engel, A. 1994, A\&AS, 104, 79

Cassatella, A., Barbero, J., \& Benvenuti, P. 1985, A\&A, 144, 335

de Kool, M., \& Ritter, H. 1993, A\&A, 267, 397

Downes, R., Webbink, R. F., \& Shara, M. M. 1997, PASP, 109, 345

Duerbeck, H. W. 1987, Space Sci. Rev., 45, 1

Duerbeck, H. W., \& Seitter, W. C. 1979, The Messenger, 17, 1

Garhart, M. P., Smith, M. A., Turnrose, B. E., Levay, K. L., \& Thompson, R. W. 1997, IUE NASA Newsletter, 57, 1

Gilmozzi, R., Selvelli, P., \& Cassatella, A. 1998, in Ultraviolet Astrophysics Beyond the Final Archive, ESA-SP, 413, 415

González Delgado, R. M., \& Pérez, E. 2000, MNRAS, 317, 64

Gonzalez-Riestra, R., Cassatella, A., \& Wamsteker, W. 2001, A\&A 373, 730

Guinan, E. F. 1990, in Evolution in Astrophysics, ESA-SP, 310, 73

Hawley, S. L. 1993, PASP, 105, 955

Henry, T. J., \& Mc Carthy, D. W. 1990, ApJ, 350, 334

Henry, T. J., \& Mc Carthy, D. W. 1993, AJ, 106, 773

Katsova, M. M., Drake, J. J., \& Livshits, M. A. 1996, in Astrophysics in the EUV, ed. S. Bowyer, \& R. F. Malina (Kluwer Acad. publ.), IAU Coll., 152 175

Kemp, J., Shambrook, A., Patterson, J., et al. 1998, PASP, 110, 380

Krautter, J., Beuermann, K., Leitherer, C., et al. 1984, A\&A, 137, 307
Landolt, A. U., Vogt, N., Schaefer, B. E., et al. 1992, ApJS, 81, 321

Legget, S. K., \& Hawkins, M. R. S. 1989, MNRAS, 238, 145

Liebert, J., Cutri, R., Ferguson D. H., et al. 1987, ApJ, 316, 399

Margon, B., \& Deutsch, E. W. 1998, ApJ, 498, L61

O’Brien, T. J., \& Cohen, J. 1998, ApJ, 498, L59

Payne-Gaposchkin, C. 1957, The Galactic Novae, North Holland

Raymont, G. B., Bromage, G. E., Jomaron C. M., et al. 1993, MNRAS, 264, 219

Rodriguez-Pascual, P. M., Gonzalez-Riestra, R., Schartel, N., \& Wamsteker, W. 1999, A\&A, 139, 183

Saizar, P., \& Ferland, G. J. 1994, ApJ, 425, 755

Savage, B. D., \& Mathis, J. S. 1979, ARA\&A, 17, 73

Schaefer, B. E. 2005, ApJ, 621, L53

Schartel, N., \& Skillen, I. 1998, in Ultraviolet Astrophysics Beyond the Final Archive, ESA-SP, 413, 735

Seitter, W. C. 1986, in RS Ophiuchi, ed. M. F. Bode (VNU Press), 63

Selvelli, P., \& Friedjung, M. 2003, A\&A, 401, 297

Selvelli, P., \& Gilmozzi, R. 2006, in preparation (Paper II)

Shabhaz, T., Livio, M., Southwell, M., \& Charles, P. A. 1997, ApJ, 484, L59

Shara, M. M., Moffat, A. F. J., Williams, R. E., \& Cohen, J. C. 1989, ApJ, 337, 720

Shara, M. M., Zurek, D. R., Williams, R. E., et al. 1997, AJ, 114, 258

Shore, S. N., Sonneborn, G., Starrfield, S., Riestra-Gonzalez, R., \& Ake, T. B. 1993, AJ, 106, 2408

Smith, D. A., \& Dhillon, V. S. 1998, MNRAS, 301, 767

Szkody, P. 1994, AJ, 108, 639

Szkody, P., \& Feinswog, L. 1988, ApJ, 334, 422

Urban, Z. 1988, in Eruptive Phenomena in Stars Comm. Konkoly Obs., Acad. Sci., 86, 359

Vennes, S., Thorstensen, J. R., Thejll, P., \& Shipman, H. L. 1991, ApJ, 372, L37 Vogt, N., Barrera, L. H., Barwig, H., \& Mantel, K. H. 1989, in AccretionPowered Compact Binaries, ed. C. W. Mauche (Cambridge Univ. Press), 391

Wade, R. A., \& Hubeny, I. 1998, ApJ, 509, 350

Warner, B. 1995, in Cataclysmic Variable Stars (Cambridge Univ. Press)

Webbink, R. F., Livio, M., Truran, J. W., \& Orio, M. (WLTO) 1987, ApJ, 314, 653

Weight, A., Evans, A., Naylor, T., Wood, J., \& Bode, M. F. 1994, MNRAS, 266, 761

Williams, R. E., Hamuy, M., \& Phillips, M. M. 1991, ApJ, 376, 721

Young, A., Skumanich, A., \& Paylor, V. 1988 ApJ, 334, 397

Young, A., Rotler, L., Skumanich, A., et al. 1991, ApJ, 378, L25 